\title{
Preliminary Study on Mechanical Properties of 3D Printed Multi- materials ABS/PC Parts: Effect of Printing Parameters
}

\author{
Pui-Voon Yap, ${ }^{1}$ Ming-Yeng Chan, ${ }^{2 *}$ and Seong-Chun Koay ${ }^{3}$ \\ ${ }^{1}$ Centre for Engineering Programmes, Imperium International College, 6.01, Level 1-7, \\ Kompleks Metro Pudu 1, Jalan Metro Pudu 2, Fraser Business Park, \\ 55200 Kuala Lumpur, Malaysia \\ ${ }^{2}$ Faculty of Engineering and Technology, Tunku Abdul Rahman University College, \\ Jalan Genting Kelang, Setapak, 53300 Kuala Lumpur, Malaysia \\ ${ }^{3}$ School of Computer Science and Engineering, Faculty of Innovation and Technology, \\ Taylor's University Lakeside Campus, No. 1, Jalan Taylor's, 47500 Subang Jaya, \\ Selangor, Malaysia
}

*Corresponding author: chanmingyeng@hotmail.my

Published online: 25 August 2021

To cite this article: Yap, P-V., Chan, M-Y. \& Koay S-C. (2021). Preliminary study on mechanical properties of 3D printed multi-materials $\mathrm{ABS} / \mathrm{PC}$ parts: Effect of printing parameters. J. Phys. Sci., 32(2), 87-104. https://doi.org/10.21315/jps2021.32.2.7

To link to this article: https://doi.org/10.21315/jps2021.32.2.7

\begin{abstract}
This research work highlights the mechanical properties of multi-material by fused deposition modelling (FDM). The specimens for tensile and flexural test have been printed using polycarbonate (PC) material at different combinations of printing parameters. The effects of varied printing speed, infill density and nozzle diameter on the mechanical properties of specimens have been investigated. Multi-material specimens were fabricated with acrylonitrile butadiene styrene (ABS) as the base material and PC as the reinforced material at the optimum printing parameter combination. The specimens were then subjected to mechanical testing to observe their tensile strength, Young's modulus, percentage elongation, flexural strength and flexural modulus. The outcome of replacing half of $A B S$ with $P C$ to create a multi-material part has been examined. As demonstrated by the results, the optimum combination of printing parameters is $60 \mathrm{~mm} / \mathrm{s}$ printing speed, $15 \%$ infill density and $0.8 \mathrm{~mm}$ nozzle diameter. The combination of ABS and PC materials as reinforcing material has improved the tensile strength (by $38.46 \%$ ), Young's modulus (by 23.40\%), flexural strength (by 23.90\%) and flexural modulus (by $37.33 \%$ ) while reducing the ductility by $14.31 \%$ as compared to pure ABS. The results have been supported by data and graphs of the analysed specimens.
\end{abstract}

Keywords: Fused deposition modelling, multi-materials 3D printed part, acrylonitrile butadiene styrene, polycarbonate, mechanical properties 


\section{INTRODUCTION}

Three-dimensional (3D) printing, which is also known as additive manufacturing (AM), is the fabrication of physical objects from a digital model by successively adding material layer by layer. ${ }^{1}$ Some of the reasons behind AM industry's rapid growth include its ability to create complex and detailed 3D objects from various types of materials. As the AM process is computer-controlled, it only requires low expertise level of operator and minimal human interaction. AM also produces minimal waste which helps reduce material and energy consumption. ${ }^{2}$

According to ISO/ASTM 52900 standard, the AM technology is divided into seven categories including binder jetting, directed energy deposition, material extrusion, material jetting, powder bed fusion, sheet lamination and vat photopolymerisation. ${ }^{3}$ A statistic from Statista has shown that the top three most popular AM technologies in 2018 are material extrusion, vat photopolymerisation which uses a light source and powder bed fusion which uses thermal energy source. ${ }^{4}$

Fused Deposition Modelling (FDM) or Fused Filament Fabrication (FFF) is the most commonly used 3D printer for fabricating polymer products in AM technology due to its low cost and its ability to achieve superior surface finishing as well as print objects of multi-material and multi-colour. Some common FDM applications include electrical enclosure, investment casting patterns, jigs and fixtures. ${ }^{5}$ The most widely used materials for FDM include polylactic acid (PLA), acrylonitrile butadiene styrene (ABS), thermoplastic polyurethane (TPU), polycarbonate (PC) and more.

FDM is one of the few AM technologies that is able to fabricate a multi-material part by using more than one extrusion nozzle. In the recent past, many studies on the properties of metal or non-metal reinforced thermoplastics have been conducted. For example, studies on reinforcement of ABS and polyamide 6 (PA6) with banana fibres, of polymer materials with metal powder and more. ${ }^{6}$ In the textile industry, attempts to fabricate garments by FDM technology have been made, but $3 \mathrm{D}$ printing materials lack the mechanical properties that allow them to replace common textile production methods like weaving and knitting. Hence, FDM is utilised to fabricate multi-material parts by combining pure 3D printing materials with textile fabrics. This ensures the enhancement of mechanical properties of the final product. ${ }^{?}$

Furthermore, there are multiple methods to fabricate a multi-material object through FDM. One of them is single nozzle printing, where the multi-material object is printed by extruding and depositing different materials through the single, same nozzle only. During the printing process, the nozzle temperature will either be the 
same for all different materials or be in a close range. This is to ensure the different materials are bonded to each other effectively and to prevent contamination of the materials. As for advantages, this method can avoid calibration defect when depositing the material at each layer. ${ }^{8}$ In order to print out a high-quality object using this method, the model has to be accurately divided into parts and then at different printing stages, the machine has to be paused to change the filament. ${ }^{9}$ There is little study of the properties of $3 \mathrm{D}$ printed multi-materials parts that printed by single nozzle printing.

The printing parameters have a huge influence on the quality and mechanical properties of the final parts. According to International Tolerance (IT) Grades, small dimensions are less accurate than large dimensions. However, using filament and extrusion nozzle of a smaller diameter can improve the accuracy of dimensionally small features. ${ }^{10}$ The mechanical properties vary according to the material used and are influenced by many factors such as infill pattern, printing speed, nozzle diameter and more.

$\mathrm{PC}$ is known for its outstanding mechanical properties and its tensile strength is ranked the second highest among all FDM materials. Besides, it has a high heat deflection temperature of $138^{\circ} \mathrm{C}$ and is commonly used for tough applications like functional testing, tooling and production. ${ }^{11}$ Meanwhile, ABS is the most widely used thermoplastic in FDM machines because of its impressive heat resistance, impact resistance, toughness, flexural strength and low thermal conductivity. ${ }^{6}$ These two thermoplastics were selected for the multi-material 3D printing via FDM. This research project is focused on the preparation of 3D printed specimens using FDM with different processing parameters. The final product, 3D printed ABS/ PC was composed of one layer of each material and it was expected to possess the advantages of both polymers. Studies were conducted to examine the mechanical properties of the final product as well as the effects of printing parameters on the mechanical properties.

\section{EXPERIMENTAL}

\subsection{Materials}

The ABS and PC 3D filaments are supplied by Fabbxible Sdn. Bhd., Pulau Pinang, Malaysia. The diameter and printing temperature of ABS and PC filaments are listed in Table 1. 
Table 1: Diameter and printing temperature of 3D ABS and PC filaments.

\begin{tabular}{lcc}
\hline Printing temperature & ABS filament & PC filament \\
\hline Diameter & $1.75 \mathrm{~mm} \pm 0.05 \mathrm{~mm}$ & $1.75 \mathrm{~mm} \pm 0.05 \mathrm{~mm}$ \\
Printing temperature & $230^{\circ} \mathrm{C}-240^{\circ} \mathrm{C}$ & $230^{\circ} \mathrm{C}-270^{\circ} \mathrm{C}$ \\
Bed temperature & $80^{\circ} \mathrm{C}-110^{\circ} \mathrm{C}$ & $80^{\circ} \mathrm{C}-110^{\circ} \mathrm{C}$ \\
Glass transition temperature & $105^{\circ} \mathrm{C}$ & $150^{\circ} \mathrm{C}$ \\
\hline
\end{tabular}

\subsection{Preparation of ABS and PC Specimens}

3D files of dumbbell-shaped and rectangular specimens were created using the computer-aided design (CAD) application, Autodesk Inventor and they were saved in stereolithography (STL) file format. Figure 1 shows overall preparation process of 3D printed specimens. The ABS/PC (50:50) specimen is shown in Figure 2.

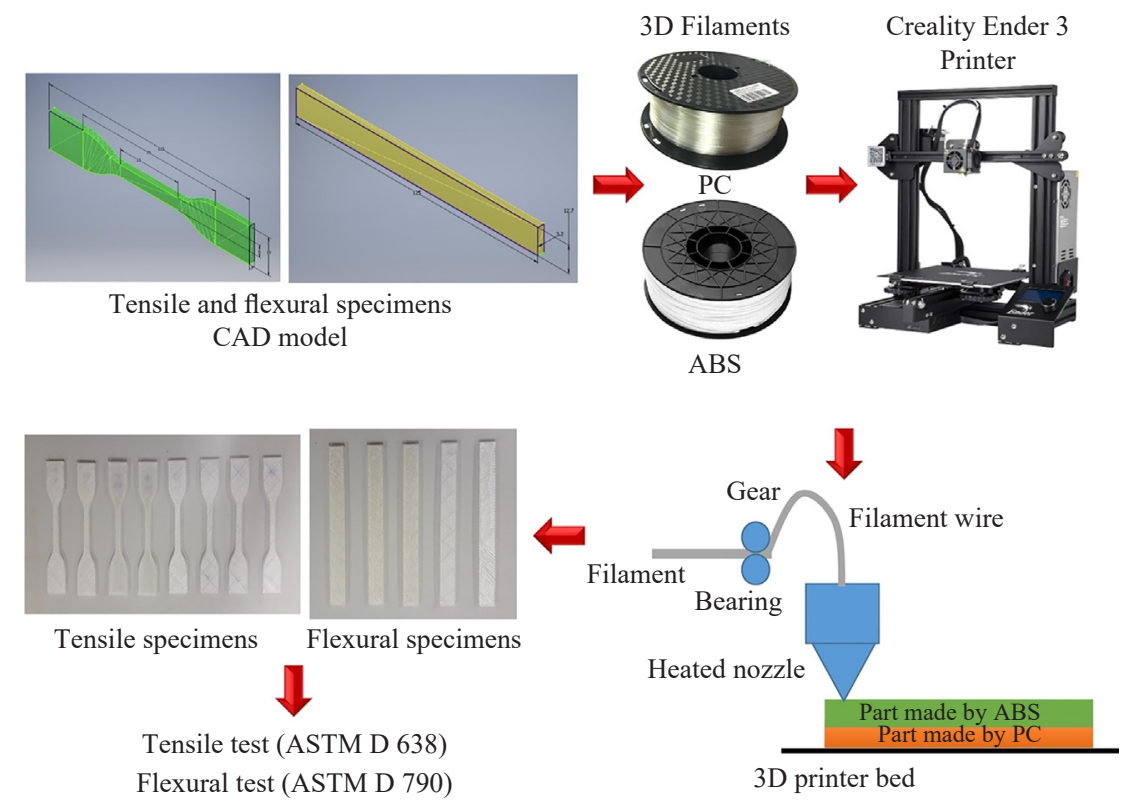

Figure 1: Overall preparation process of 3D printed specimens.

Using the 3D printer slicing application, Creality Ender 3 software, the print settings such as layer height, infill pattern, infill density, nozzle diameter, printing speed, temperature and more can be easily adjusted. The $3 \mathrm{D}$ printing process also can be simulated in the preview stage before the 3D file is sliced into hundreds of horizontal layers. A new file format called G-code was then generated and fed into the $3 \mathrm{D}$ printer in order to print out the specimen. 


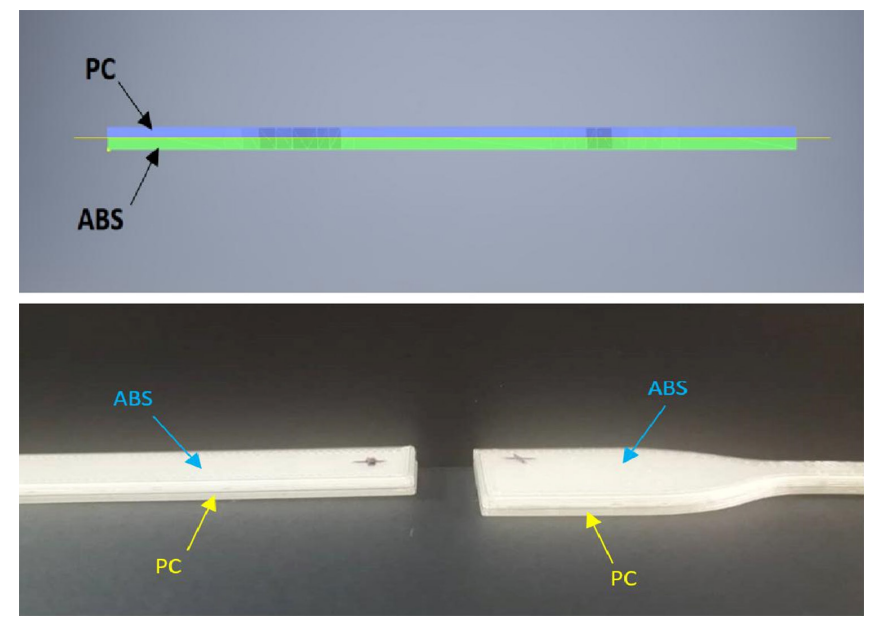

Figure 2: $\mathrm{ABS} / \mathrm{PC}$ specimen.

Creality Ender 3 printer with one extrusion nozzle was used to fabricate the specimens. When printing the ABS/PC (50:50) specimens, the printing process must be paused halfway in order to switch the filament. Then, the printing was resumed. Every piece of the $3 \mathrm{D}$ printed product was examined to make sure that there was no warping and every layer was adhered well to the layer beneath it. A set of 10 specimens were printed for each combination of printing parameters. The printing parameters are presented in Table 2.

Table 2: Printing parameters of specimens.

\begin{tabular}{lc}
\hline Printing parameter & \\
\hline Layer height & $0.3 \mathrm{~mm}$ \\
Shell thickness & $0.8 \mathrm{~mm}$ \\
Infill pattern & $\mathrm{Grid}$ \\
Extruder temperature & $240^{\circ} \mathrm{C}$ \\
Print bed temperature & $100^{\circ} \mathrm{C}$ \\
Diameter of nozzle & $\varnothing 0.4 \mathrm{~mm}, \varnothing 0.6 \mathrm{~mm}, \varnothing 0.8 \mathrm{~mm}$ \\
Printing speed & $60 \mathrm{~mm} / \mathrm{s}, 80 \mathrm{~mm} / \mathrm{s}, 100 \mathrm{~mm} / \mathrm{s}$ \\
Infill density & $10 \%, 15 \%, 20 \%$ \\
\hline
\end{tabular}

\subsection{Mechanical Test}

Tensile and flexural specimens were designed with the help of CAD, and then the drawing was converted to STL file format before 3D printing the specimens. The tensile and flexural tests were performed using a testing machine (Model VEW 
2302, VICTOR Material Testing Equipment, Ho Chi Minh, Vietnam). The tensile specimens were printed in dumbbell shape, (according to ASTM D638 standard), while flexural specimens were printed in rectangular shape, (according to ASTM D790 standard). Both of the tests were carried out at room temperature with $10 \mathrm{kN}$ of load was applied on the specimens. Moreover, a cross-head speed of $15 \mathrm{~mm} / \mathrm{min}$ was used. There are at least 10 specimens were tested with each processing parameter in order to get an average value.

\subsection{Statistical Analysis}

Single factor analysis of variance (ANOVA) from Microsoft Excel 2013 was used to determine the significance between the means data of single and multi-materials 3D printed parts. The difference between means was considered significant when $P \leq 0.05$.

\section{RESULTS AND DISCUSSION}

\subsection{PC Specimens with Varied Printing Speeds}

The specimens were printed with varied printing speeds at settings $60 \mathrm{~mm} / \mathrm{s}$, $80 \mathrm{~mm} / \mathrm{s}$ and $100 \mathrm{~mm} / \mathrm{s}$ for tensile and flexural tests respectively. Meanwhile, the infill density of $20 \%$ and nozzle diameter of $0.4 \mathrm{~mm}$ were fixed thoroughly. As shown in the stress-strain curves of specimens in Figure 3, specimens printed with $60 \mathrm{~mm} / \mathrm{s}$ speed have demonstrated relatively higher strength, ductility and toughness as compared to specimens printed with $80 \mathrm{~mm} / \mathrm{s}$ and $100 \mathrm{~mm} / \mathrm{s}$ speeds, respectively. The specimens printed with $100 \mathrm{~mm} / \mathrm{s}$ speed had the lowest stiffness, ductility and strength. After reaching the yield point, all specimens experienced significant plastic deformation. The increase in strengths can be observed as the specimens began to strain harden until failure. The specimens printed with $60 \mathrm{~mm} / \mathrm{s}$ speed underwent the largest plastic deformation, while the plastic deformation is the smallest for specimens printed with $100 \mathrm{~mm} / \mathrm{s}$ speed.

Figure 4 shows that the values of (a) tensile strength, (b) Young's modulus and (c) elongation at maximum stress decreased as printing speed increased. When the specimens were printed with $60 \mathrm{~mm} / \mathrm{s}$ speed, the highest mean values of tensile strength $(39 \mathrm{MPa})$, Young's modulus $(0.83 \mathrm{GPa})$ and elongation at maximum stress $(30.53 \%)$ were obtained. Meanwhile, the second highest tensile strength (34 MPa), Young's modulus $(0.76 \mathrm{GPa})$ and percentage elongation $(28.48 \%)$ were acquired from printing at $80 \mathrm{~mm} / \mathrm{s}$ speed. The specimens printed with speed of $100 \mathrm{~mm} / \mathrm{s}$ show the lowest average tensile strength (31 MPa), Young's modulus (0.66 GPa) and elongation at maximum stress $(27.27 \%)$. 


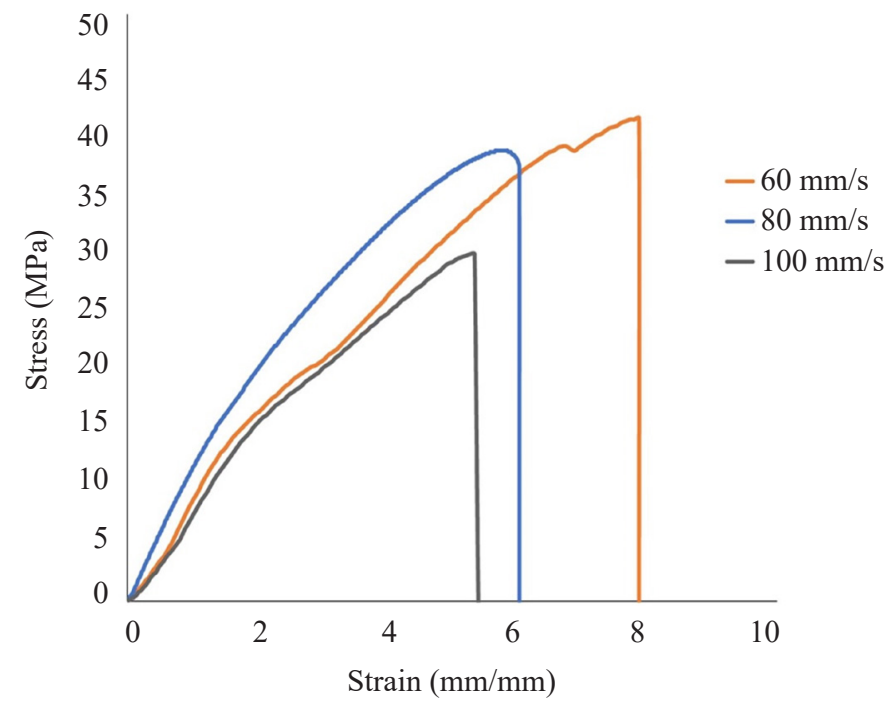

Figure 3: Stress-strain curves of specimens with varied printing speeds.

(a)

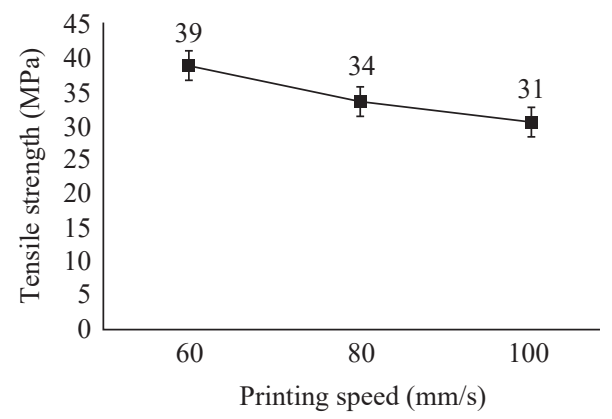

(b)

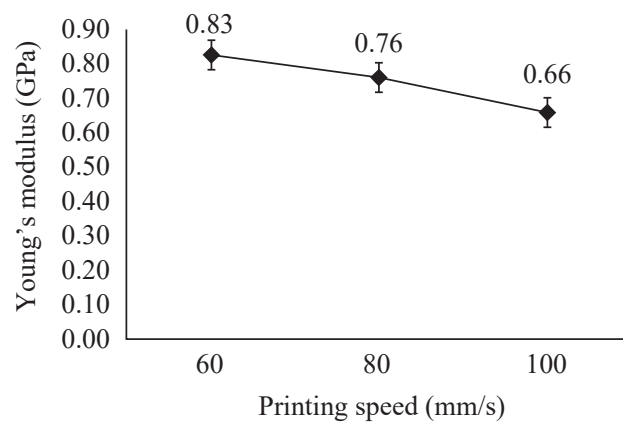

(c)

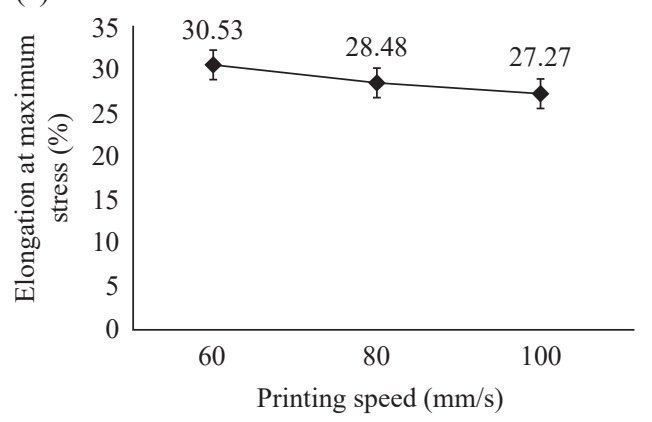

Figure 4: (a) Tensile strength, (b) Young's modulus and (c) elongation at maximum stress with different printing speeds. 
Figure 5 shows the graphs of flexural strength and flexural modulus against printing speed. It can be seen that the flexural strength $(28 \mathrm{MPa})$ and flexural modulus $(16.72 \mathrm{GPa}$ ) were the highest for the set printed with $60 \mathrm{~mm} / \mathrm{s}$ speed. As the printing speed increased from $80 \mathrm{~mm} / \mathrm{s}$ to $100 \mathrm{~mm} / \mathrm{s}$, there were small decrements in both flexural strength (from $18 \mathrm{MPa}$ to $12 \mathrm{MPa}$ ) and flexural modulus (from $12.68 \mathrm{GPa}$ to $10.33 \mathrm{GPa}$ ).

(a)

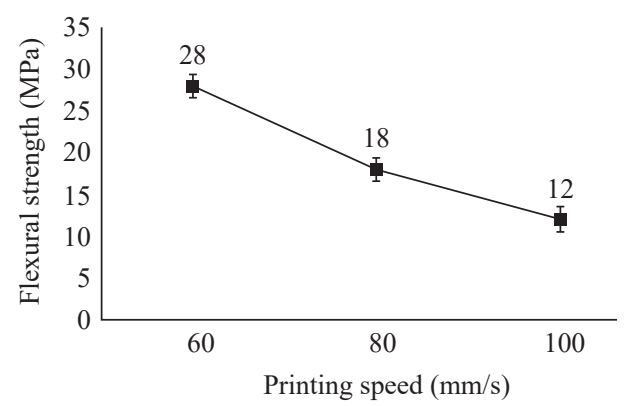

(b)

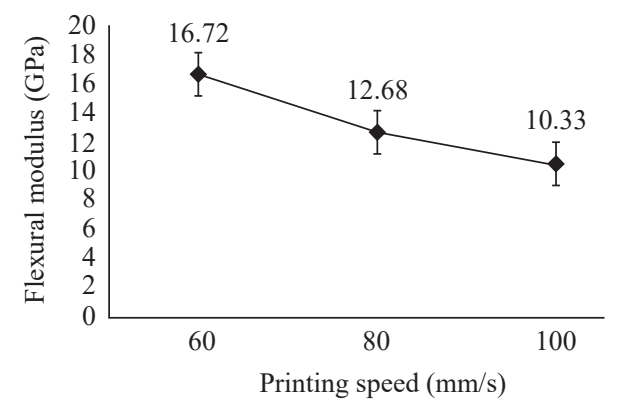

Figure 5: (a) Flexural strength and (b) flexural modulus against printing speed.

The reduction of printing speed from $100 \mathrm{~mm} / \mathrm{s}$ to $60 \mathrm{~mm} / \mathrm{s}$ has caused rises in tensile strength (by 25.81\%), Young's modulus (by 25.76\%), percentage elongation (by 11.95\%), flexural strength (by 133\%) and in flexural modulus (by $61.18 \%$ ). This shows that printing with lower speed has resulted in better mechanical and flexural properties such as higher strengths, stiffness and ductility. Printing at lower speed could allow the filament to be completely melted before being deposited, ensuring stronger interlayer bond, and consistency in the extrusion process. It had also prevented major print defects that could be caused when printing at a high speed. According to Chennakesava and Narayan, the mechanical properties of an object are affected directly by the bonding of filaments. ${ }^{12}$ It was concluded from this experiment that printing setting at $60 \mathrm{~mm} / \mathrm{s}$ is the optimum printing speed. Hence, all the specimens for the remaining experiments were printed with this speed setting.

A study conducted by Sukindar et al. also proven that the specimen printed with the highest speed has the lowest tensile strength. ${ }^{13}$ This is because when printing with high speed, the temperature of the filament inside the nozzle is unable to remain stable. As the nozzle moves rapidly across the print bed, the filament is deposited regardless if it has melted completely or not. This will then lead to inconsistency of the extrusion process and produce print defects. Therefore, the most ideal printing speed should be set lower with consideration of the printing duration. 


\subsection{PC Specimens with Varied Infill Densities}

The parameter combination for this experiment was printing speed of $60 \mathrm{~mm} / \mathrm{s}$, nozzle diameter of $0.4 \mathrm{~mm}$, and varied infill densities of $10 \%, 15 \%$ and $20 \%$, respectively. Figure 6 shows the stress-strain curves of specimens printed with different infill densities. Results revealed that specimens printed with $15 \%$ infill density have achieved the highest strengths, toughness and ductility in comparison with specimens printed with $10 \%$ and $20 \%$ infill densities. Specimens printed with $20 \%$ infill density have exhibited the lowest stiffness, toughness and strength among all specimens. All specimens experienced noticeable plastic deformation and their strengths continued to rise as strain hardening occurred. Upon reaching the ultimate strength points, their strengths decreased as necking occurred before the specimens failed. As compared to specimens printed with $10 \%$ and $20 \%$ infill densities, the specimens with $15 \%$ underwent the strongest plastic deformation.

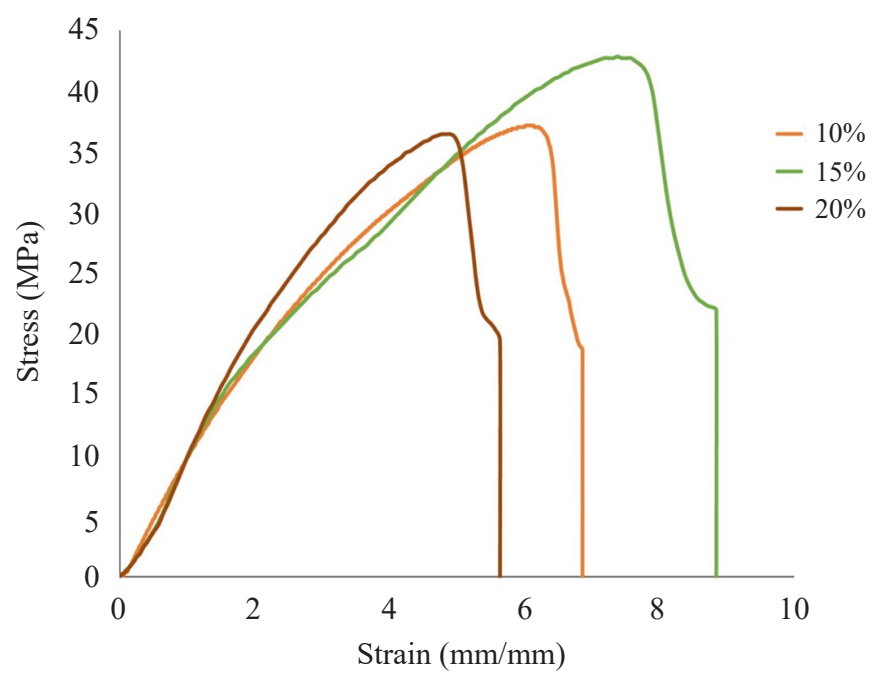

Figure 6: Stress-strain curves of specimens with varied infill densities.

Figure 7 shows that the graphs of (a) tensile strength, (b) Young's modulus and (c) elongation at maximum stress against infill density. It is observed that the set of specimens printed with $15 \%$ infill density has obtained the highest tensile strength (43 MPa) and Young's modulus ( $0.68 \mathrm{GPa})$. However, as can be seen in Figure 7 , its percentage elongation is only the second highest at $30.34 \%$. The greatest elongation of $35.86 \%$ could be found at $10 \%$ infill density, which had the second highest tensile strength at $38 \mathrm{MPa}$ and Young's modulus at $0.59 \mathrm{GPa}$. The average tensile strength (35 MPa), Young's modulus $(0.45 \mathrm{GPa})$ and percentage elongation $(26.17 \%)$ were the lowest for the set printed with $20 \%$ infill density. 
(a)

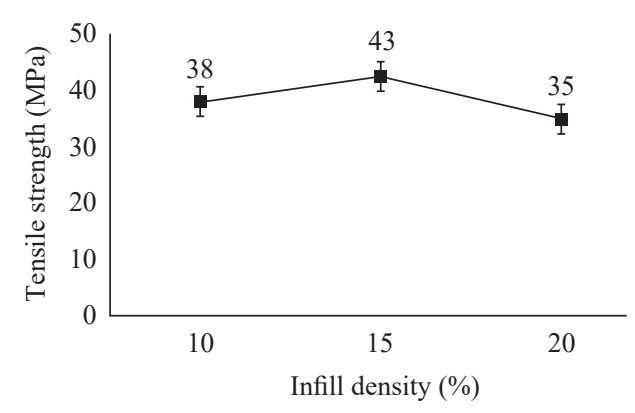

(b)

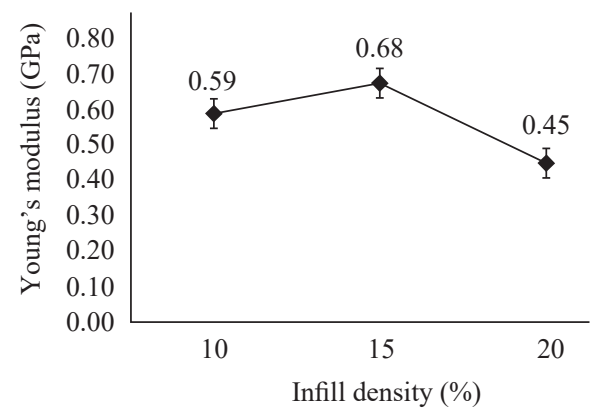

(c)

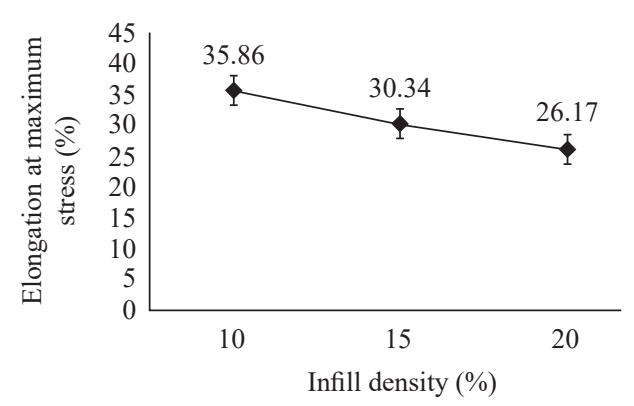

Figure 7: (a) Tensile strength, (b) Young's modulus and (c) elongation at maximum stress with different infill densities.

As shown in Figure 8 , the set printed with $20 \%$ infill density had achieved the highest flexural strength at $26 \mathrm{MPa}$ and flexural modulus at $16.67 \mathrm{GPa}$. As the infill densities reduced from $15 \%$ to $10 \%$, the flexural strength had decreased from $24 \mathrm{MPa}$ to $20 \mathrm{MPa}$. The flexural modulus also dropped from $9.16 \mathrm{GPa}$ to $5.16 \mathrm{GPa}$.

Based on this experiment, printing with 15\% infill density has increased the tensile strength by $11.63 \%$ than printing with $20 \%$ infill density. It has also boosted the Young's modulus by $15.25 \%$ and elongation at maximum stress by $15.39 \%$. The flexural strength and flexural modulus, however, were reduced by $8.33 \%$ and $45.05 \%$, respectively when the infill density was lowered from $20 \%$ to $15 \%$.

However, studies have shown that printing with a lower infill density can cause more air gaps between filament threads and result in lower strength. It is also understandable that every specimen can always respond differently as it was 
composed of multiple layers consisting with multiple threads. Overall, the best result was achieved when the specimens were printed with $15 \%$ infill density. This parameter was then set as a constant for the remaining number of experiments.

(a)

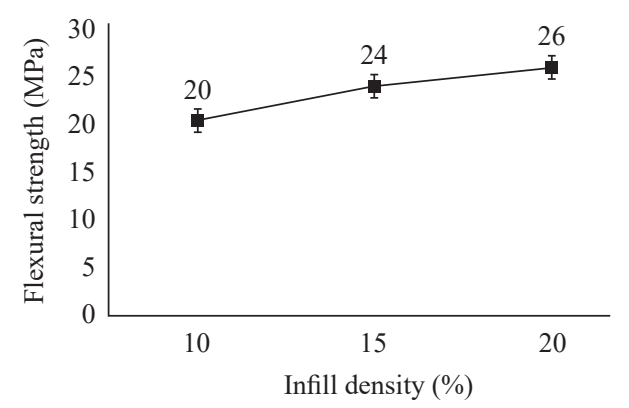

(b)

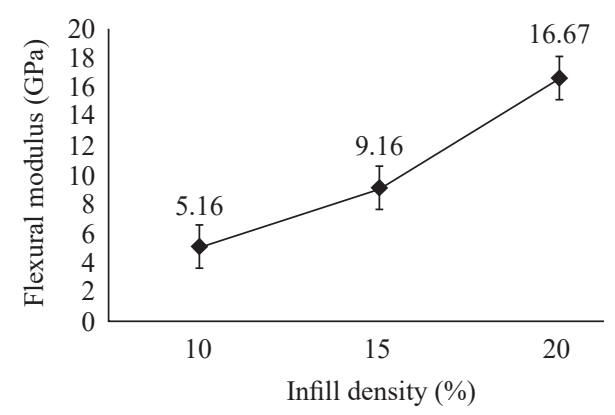

Figure 8: (a) Flexural strength and (b) flexural modulus against infill densities.

According to Terekhina et al., the researches have shown that a higher infill density can increase a printed part's strength, ductility and capacity to absorb energy. ${ }^{14}$ This is due to printing with higher infill density can form more continuous layers and hence this part will be less porous and stronger than the former. However, the optimum infill density should depend solely on the object. For objects that are not designed to support load, a 10\% infill density is recommended. An infill density of $20 \%$ will be sufficient for an object to hold small load. This is also the standard setting to fabricate an object with great strength and low weight in a relatively short time. For an object that requires greater resistance and strength, an infill density of $60 \%$ or above is ideal. ${ }^{15}$

\subsection{PC Specimens with Varied Nozzle Diameters}

For this experiment, the printing speed and infill density were set as $60 \mathrm{~mm} / \mathrm{s}$ and $15 \%$, respectively, which results obtained from previous experiment. As observed from the tensile stress-strain curves in Figure 9, the set of specimens printed with $0.8 \mathrm{~mm}$ nozzle diameter exhibited the highest strengths and stiffness. It also indicated that they have experienced the largest plastic deformation before rupture and hence, exhibiting the highest ductility and toughness. In comparison, the specimens printed with $0.4 \mathrm{~mm}$ nozzle diameter had the lowest strength, toughness and ductility. After reaching the ultimate tensile strength point, necking occurred before the specimens failed. This set of specimens also have experienced the smallest plastic deformation. 


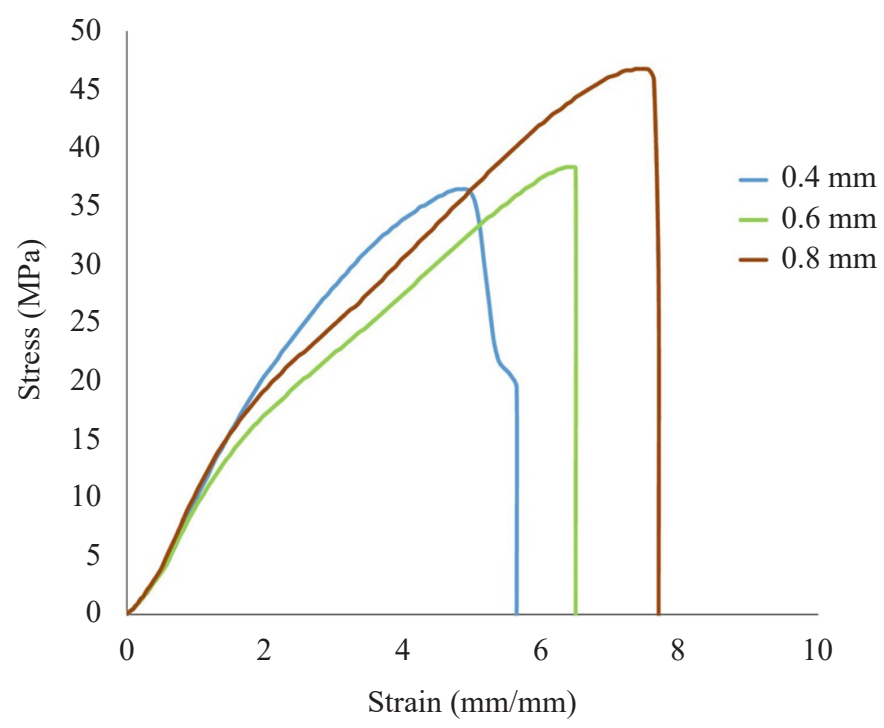

Figure 9: Stress-strain curves of specimens with varied nozzle diameter.

As evident in Figure 10, printing with $0.8 \mathrm{~mm}$ nozzle diameter has provided the highest tensile strength at $46 \mathrm{MPa}$, Young's modulus at $0.63 \mathrm{GPa}$ and percentage elongation at $31.79 \%$. The second highest tensile strength, Young's modulus and percentage elongation were acquired at $0.6 \mathrm{~mm}$ nozzle diameter, which were $40 \mathrm{MPa}, 0.57 \mathrm{GPa}$ and $31.79 \%$, respectively. Printing with the standard $0.4 \mathrm{~mm}$ nozzle diameter, however, has resulted in the lowest tensile strength at $37 \mathrm{MPa}$, Young's modulus at $0.49 \mathrm{GPa}$ and percentage elongation at $29.77 \%$.

Figure 11 graphs show that increment in nozzle diameter has also resulted in increment in flexural strength and flexural modulus. The nozzle diameter of $0.8 \mathrm{~mm}$ has attributed to the highest flexural strength at $45 \mathrm{MPa}$ and flexural modulus at $21.08 \mathrm{GPa}$. It was followed by flexural strength of $38 \mathrm{MPa}$ and modulus of $16.60 \mathrm{GPa}$ at $0.6 \mathrm{~mm}$ nozzle diameter. Printing with $0.4 \mathrm{~mm}$ nozzle diameter has once again obtained the lowest flexural strength and flexural modulus at $26 \mathrm{MPa}$ and $13.73 \mathrm{GPa}$, respectively.

As a comparison between printing with $0.4 \mathrm{~mm}$ nozzle diameter and $0.8 \mathrm{~mm}$ nozzle diameter, it can be observed that the latter has caused improvements in tensile strength by $24.32 \%$, Young's modulus by $28.57 \%$, percentage elongation by $6.78 \%$, flexural strength by $73.08 \%$ and flexural modulus by $34.87 \%$. Hence, it can be gathered that printing with a nozzle of $0.8 \mathrm{~mm}$ diameter has achieved the best mechanical and flexural properties. 
(a)

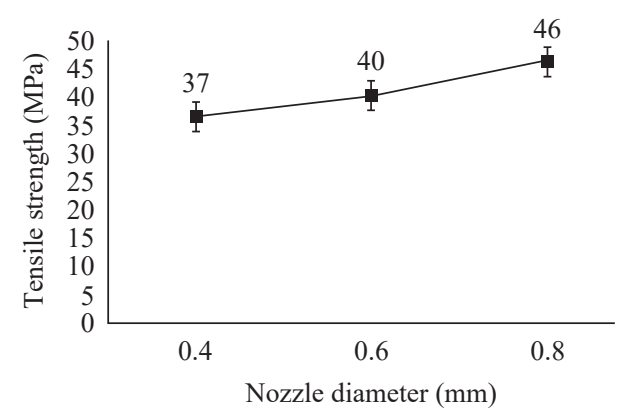

(b)

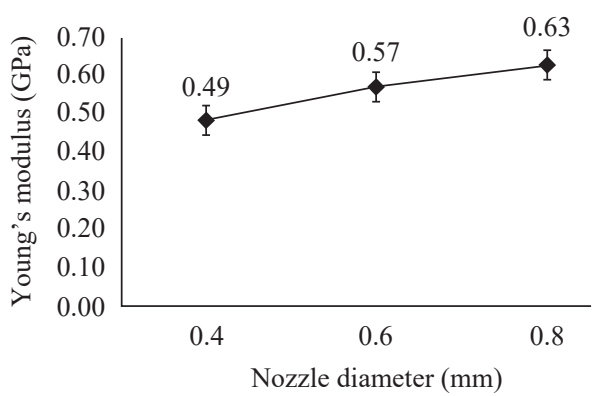

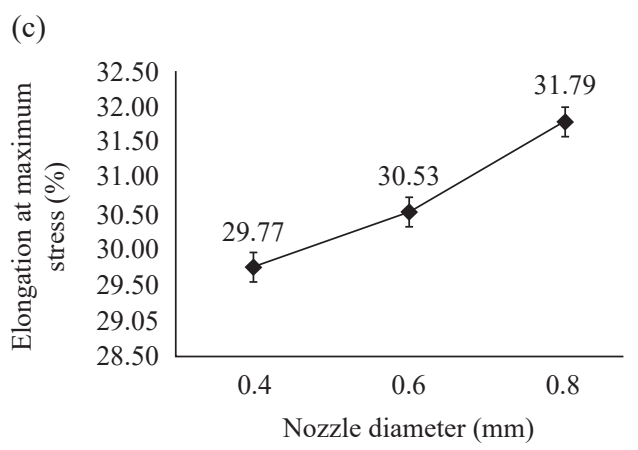

Figure 10: (a) Tensile strength, (b) Young's modulus and (c) elongation at maximum stress with different nozzle diameter.

(a)

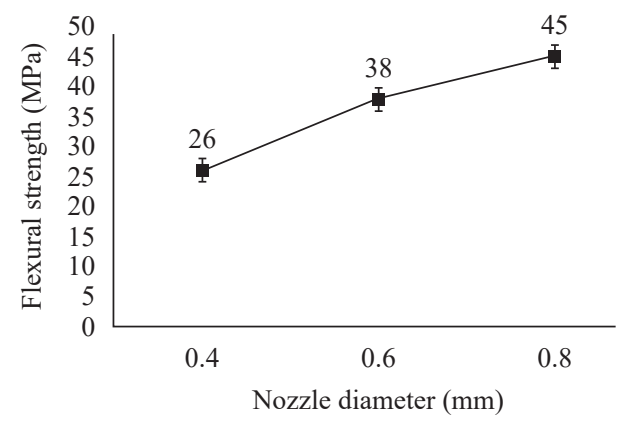

(b)

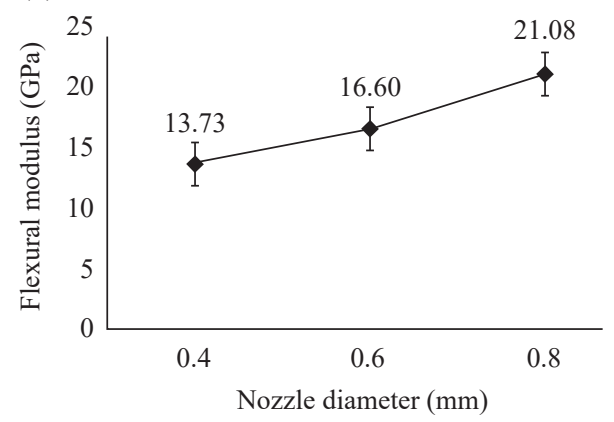

Figure 11: (a) Flexural strength and (b) flexural modulus against nozzle diameter.

Although using a larger nozzle can worsen a printed object's accuracy and intricacy, it can improve the toughness of the object. This is due to nozzle with larger diameter can extrude filament in thicker threads and thus, creating a more durable and sturdier object. Wang et al. reported that the extrusion through a 
nozzle with small diameter is able to improve the accuracy and intricacy of the finished object. ${ }^{16}$ However, a nozzle that is too small will be blocked easily. A nozzle with larger diameter can extrude filament with in thicker layers or strings and hence, reducing the printing time effectively. Printing with thicker strings can also improve the adhesion between the first layer of material and the print bed. Even so, it is unfavourable in terms of surface quality.

It was revealed from these three experiments studying the effects of printing speed, infill density and nozzle diameter on the mechanical properties of printed objects that the optimum values obtained were $60 \mathrm{~mm} / \mathrm{s}, 15 \%$ and $0.8 \mathrm{~mm}$. Accordingly, the $\mathrm{ABS}$ and $\mathrm{ABS} / \mathrm{PC}$ specimens for the next experiment were printed with these parameter values.

\subsection{Mechanical Properties of 3D Printed Single PC, ABS and Multi- materials ABS/PC Specimens}

As can be observed from the stress-strain curves in Figure 12, the pure PC specimens had the highest stiffness, strength, toughness and ductility. The combination of $\mathrm{PC}$ and $\mathrm{ABS}$ materials has resulted in higher strength and stiffness than pure ABS specimens as well as higher flexibility than pure PC specimens. However, it was noted that the addition of PC material has caused reduction in ductility as multi-material $\mathrm{ABS} / \mathrm{PC}$ specimens experienced smaller plastic deformation than pure ABS specimens.

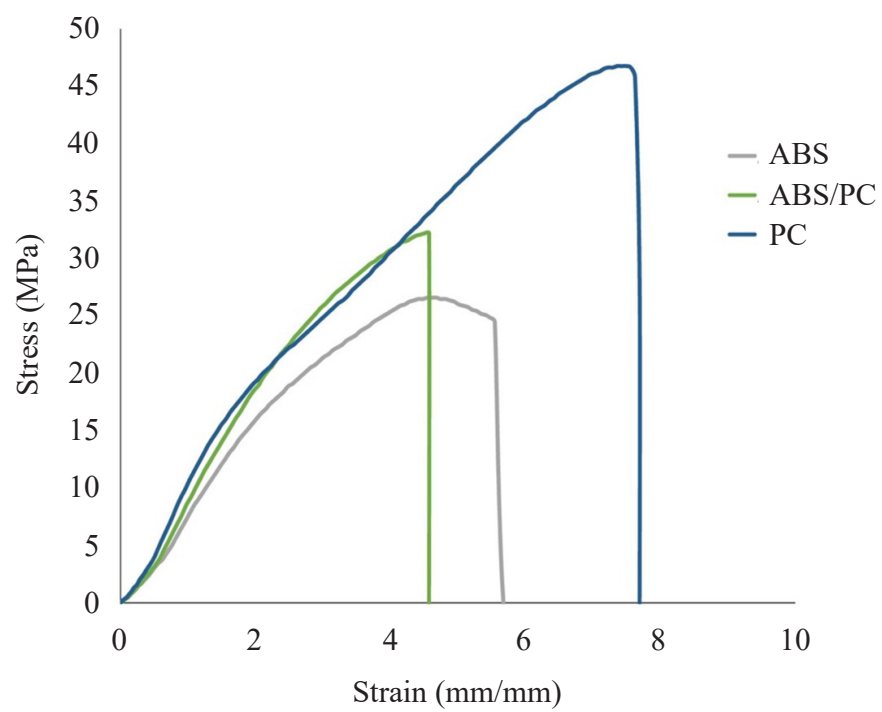

Figure 12: Stress-strain curves of $3 \mathrm{D}$ printed $\mathrm{ABS}, \mathrm{PC}$ and multi-material $\mathrm{ABS} / \mathrm{PC}$ parts. 
As demonstrated in Figure 13, the add-on of PC material has significantly increased $(P<0.05)$ the tensile strength of ABS specimens from $26 \mathrm{MPa}$ to $36 \mathrm{MPa}$. The addition of $\mathrm{PC}$ material $(\mathrm{P} \approx 0.05)$ increased the Young's modulus from $0.94 \mathrm{GPa}$ to $1.16 \mathrm{GPa}$. Meanwhile, the average elongation at maximum stress of $\mathrm{ABS} / \mathrm{PC}$ specimens was $14.31 \%$ lower than that of pure ABS specimens.

(a)

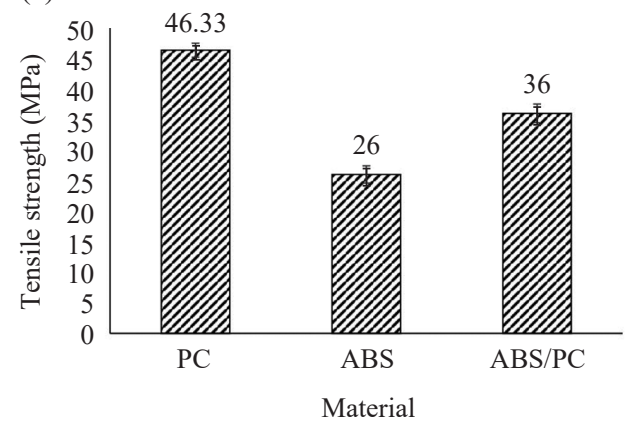

(c)

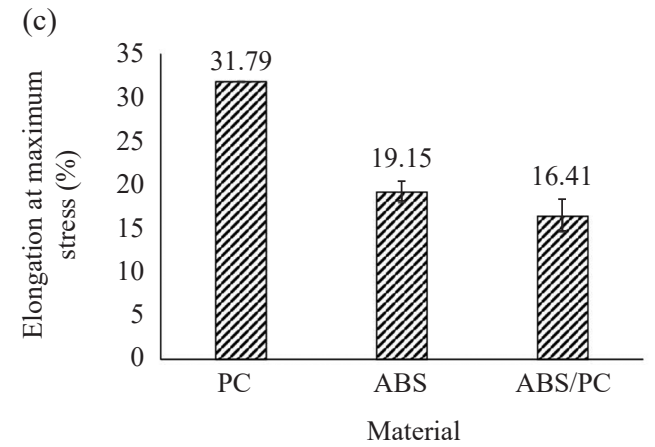

(b)

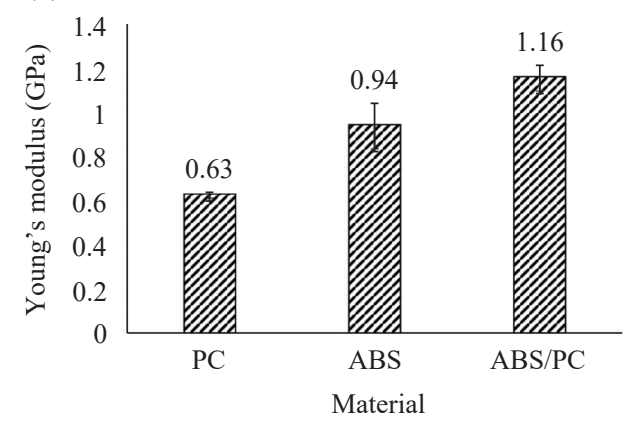

Figure 13: (a) Tensile strength, (b) Young's modulus, and (c) elongation at maximum stress of $3 \mathrm{D}$ printed $\mathrm{ABS}, \mathrm{PC}$ and multi-material $\mathrm{ABS} / \mathrm{PC}$ parts.

The graphs of flexural strength and flexural modulus in Figure 14 shows that printed $\mathrm{ABS} / \mathrm{PC}$ specimens have obtained flexural strength of $38 \mathrm{MPa}$ and flexural modulus of $15.78 \mathrm{GPa}$, which are higher than $30.67 \mathrm{MPa}$ flexural strength and $9.89 \mathrm{GPa}$ flexural modulus of pure ABS specimens. Hence, the addition of $\mathrm{PC}(\mathrm{P} \approx 0.05)$ has significantly enhanced the flexural strength and flexural modulus of $3 \mathrm{D}$ printed $\mathrm{ABS} / \mathrm{PC}$ parts.

It can be summarised from this experiment that by adding PC material to ABS specimens has improved the tensile strength by $38.46 \%$, modulus of elasticity by $23.40 \%$, flexural strength by $23.90 \%$, and bend modulus by $37.33 \%$. However, it has also lowered the ductility by $14.31 \%$. The concept of multi-material FDM is 
similar, regardless of using thermoplastic blend or multiple materials in a sandwich structure. This method can efficiently develop new materials with improved or modified properties depending on the composition of materials used. As can be seen in this research project, the multi-material $\mathrm{ABS} / \mathrm{PC}$ specimen has possessed the high strength and flexibility, which are rendered by BPA in PC and butadiene in ABS, respectively.

(a)

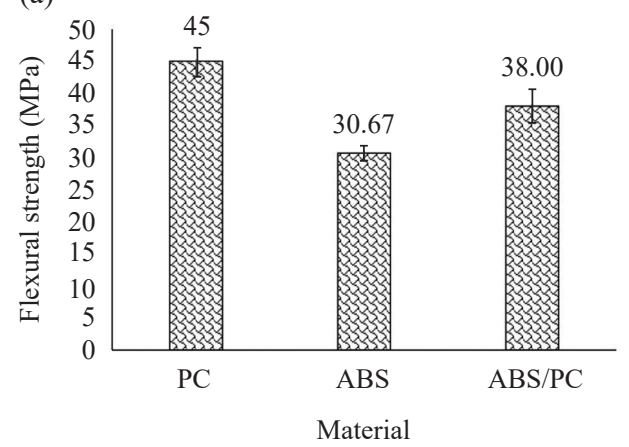

(b)

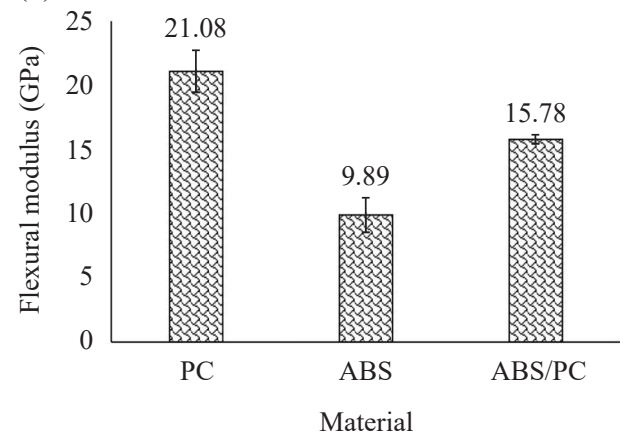

Figure 14: (a) Flexural strength and (b) flexural modulus of 3D printed ABS, PC and multi-material ABS/PC parts.

Similar finding was reported by Singh et al. ${ }^{17}$ The study was intended to fabricate a multi-material part that contains all the advantages of the three thermoplastics used: ABS, PLA and high impact polystyrene (HIPS). The specimens were subjected to tensile and pull-out tests. Among the materials, HIPS had the lowest tensile strength of $4.21 \mathrm{MPa}$ and peak strength of $27.4 \mathrm{~kg} / \mathrm{mm}^{2}$. At the best printing settings, the multi-material specimen has managed to improve the tensile and peak strengths by $6.57 \mathrm{MPa}$ and $1.41 \mathrm{~kg} / \mathrm{mm}^{2}$. The results show that multi-material FDM using ABS, PLA and HIPS, or other types of thermoplastics is doable and can improve the properties of $3 \mathrm{D}$ printed object.

\section{CONCLUSION}

This research project has studied the influences of FDM printing parameters on the mechanical properties of one of the most widely used thermoplastics in the AM industry: PC. It was learned that the ideal speed and nozzle diameter to print PC object were $60 \mathrm{~mm} / \mathrm{s}$ and $0.8 \mathrm{~mm}$, respectively. Printing results with these parameter values have shown great improvement in mechanical and flexural properties. However, printing with a slightly lower infill density $(15 \%)$ has surprisingly caused enhancements in strength, stiffness and ductility, while diminishing the flexural properties. 
The main focus of this study was to observe the mechanical properties of 3D printed multi-material part through FDM. The materials used were ABS and PC. The ABS/PC part was printed with the optimum parameter combination: $60 \mathrm{~mm} / \mathrm{s}$ printing speed, $15 \%$ infill density and $0.8 \mathrm{~mm}$ nozzle diameter, and the materials were able to adhere well during printing. It can be noted that multi-material FDM of various thermoplastics and especially of ABS and PC is achievable. Due to PC being a tough thermoplastic with magnificent strength and stiffness, it has improved the rigidity and bending stiffness of pure ABS object while lessening its ductility. In detail, the mechanical properties of $3 \mathrm{D}$ printed multi-materials ABS/PC parts have shown improvement in tensile strength by $38.46 \%$, Young's modulus by $23.40 \%$, flexural strength by $23.90 \%$ and flexural modulus by $37.33 \%$ as compared to pure ABS.

This research project has demonstrated that multi-material FDM can overcome the limitations of single material. Besides, it can widen the usage and exploration of materials. Multi-material FDM also provides flexibility in developing complex functional designs and products with improved properties.

\section{REFERENCES}

1. Shahrubudin, N., Lee, T. C. \& Ramlan, R. (2019). An overview on 3D printing technology: Technological, materials, and applications. Procedia Manuf., 35, 1296-1287. https://doi.org/10.1016/j.promfg.2019.06.089.

2. Pîrjan, A. \& Petroşanu, D. M. (2013). The impact of 3D printing technology on the society and economy. J. Inf. Syst. Operations Manag., 7(2), 360-370.

3. ISO/ASTM 52900. (2015). Additive manufacturing-General principlesTerminology. Switzerland: ISO.

4. Holst, A. (2019). Most used 3D printing technologies worldwide 2018. Retrieved 7 October 2020 from https://www.statista.com/statistics/756690/worldwide-mostused-3d-printing-technologies/

5. All3DP. (2019). The types of $3 D$ printing technology. Retrieved 8 October 2020 from https://all3dp.com/1/types-of-3d-printers-3d-printing-technology/

6. Singh, R. et al. (2019). Multi-material additive manufacturing of sustainable innovative materials and structures. Polym., 11(1), 62-75. https://doi.org/10.3390/ polym 11010062 .

7. Sabantina, L. et al. (2015). Combining 3D printed forms with textile structuresmechanical and geometrical properties of multi-material systems. IOP Conf. Ser.: Mater. Sci. Eng., 87, 012005. https://doi.org/10.1088/1757-899X/87/ 1/012005 
8. Baca, D. \& Ahmad, R. (2020). The impact on the mechanical properties of multimaterial polymers fabricated with a single mixing nozzle and multi-nozzle systems via fused deposition modelling. Inter. J. Adv. Manuf. Technol., 106, 4509-4520. https://doi.org/10.1007/s00170-020-04937-3

9. Bryll, K. et al. (2018). Polymer composite manufacturing by FDM 3D printing technology. In MATEC Web Conf., 237, 02006. https://doi.org/10.1051/ matecconf $/ 201823702006$

10. Minetola, P., Iuliano, L. \& Marchiandi, G. (2016). Benchmarking of FDM machines through part quality using IT grades. Procedia CIRP, 41, 1027-1032. https://doi.org/10.1016/j.procir.2015.12.075.

11. Fischer, F. (2011). Thermoplastics: The best choice for $3 D$ printing. Eden Prairie: Stratasys Incorporated.

12. Chennakesava, P. \& Narayan, Y. S. (2014). Fused deposition modeling - Insights. Inter. Conf. Adv. Des. Manuf. ICAD\&M, 14, 1345-1350.

13. Sukindar, N. A. B. et al. (2017). Analysis on the impact process parameters on tensile strength using 3D printer Repetier-host software. ARPN J. Eng. Appl. Sci., 12(10), 3341-3346.

14. Terekhina, S. et al. (2019). Effects of the infill density on the mechanical properties of nylon specimens made by filament fused fabrication. Technol., 7(3), 57. https://doi.org/10.3390/technologies7030057.

15. Filament2Print. (2019). The importance of the type of infill in $3 D$ printing. Retrieved 5 April 2020 from https://filament2print.com/gb/blog/71_importance -infill-3d-printing.html.

16. Wang, P. et al. (2019). Effects of printing parameters of fused deposition modeling on mechanical properties, surface quality, and microstructure of PEEK. J. Mater. Process. Technol., 271, 62-74. https://doi.org/10.1016/j.jmatprotec .2019.03.016.

17. Singh, R. et al. (2019). Multi-material additive manufacturing of sustainable innovative materials and structures. Polym., 11(1), 1-14. https://doi.org/10.3390/ polym11010062. 